\title{
Hábito de tabagismo entre adolescentes de escolas brasileiras
}

\author{
Tania Aparecida da Silva Klein \\ Doutora em Ensino de Ciências e Educação Matemática, Docente do Curso de Ciências Biológicas e \\ Coordenadora do Curso de Pós Graduação em Ensino de Ciências Biológicas da Universidade Estadual de \\ Londrina \\ $\triangle \underline{\text { taniaklein@uel.br }}$
}

Beatriz Nazo Mônaco

Discente do Curso de Licenciatura e Bacharelado em Ciências Biológicas da Universidade Estadual de Londrina. $\triangle$ beatriz.monaco@uel.br

\section{Giovanna Ivy Prado Santos}

Discente do Curso de Licenciatura e Bacharelado em Ciências Biológicas da Universidade Estadual de Londrina. $\triangle$ giovanna.prado@uel.br

\section{Bruna Alves da Silva}

Discente do Curso de Licenciatura e Bacharelado em Ciências Biológicas da Universidade Estadual de Londrina. \bruna.alves.silva@uel.br

\section{Andreia de Freitas Zômpero}

Pós-Doutora em Ensino de Ciências. Docente do curso de Ciências Biológicas e Programa de Pós-Graduação em Ensino de Ciências e Educação Matemática da Universidade Estadual de Londrina.

凶andzomp@yahoo.com.br

\begin{abstract}
Resumo:
Mesmo com a gama de informações disponíveis sobre os malefícios causados pela exposição contínua à nicotina, o tabaco ainda se encontra na segunda posição no ranking de drogas lícitas mais consumidas segundo o INCA. Esta pesquisa faz parte de um projeto maior que tem como tema central a implementação de atividades pedagógicas sobre tabagismo e adolescência, nas escolas do município de Londrina - PR, tendo como foco adolescentes com idades entre 14 e 20 anos. Os objetivos do trabalho foram identificar a causa do interesse dos jovens pelo hábito do tabagismo, assim como o interesse do adolescente fumante pelo consumo das diferentes formas do tabagismo (cigarro, narguilé e/ou cigarro eletrônico/vape) e quais as influências para o consumo do tabagismo entre adolescentes. Foi aplicado um questionário junto aos alunos do Ensino Médio, matriculados em escolas públicas da região periférica e central do município de Londrina/PR. Os resultados indicaram que 39,4\% já tiveram interesse e já experimentaram uma das três formas de tabagismo (cigarro, narguilé e/ou cigarro eletrônico/vape), além disso, o hábito de fumar narguilé foi o que mais apareceu entre os estudantes. Ressalta-se que a escola, por suas características, torna-se um ambiente de excelência para realização de práticas educativas para prevenir o consumo de cigarro entre os adolescentes.
\end{abstract}

Palavras-chave: Tabagismo, adolescência, escola, educação em saúde. 


\title{
Smoking habit among adolescents from Brazilian schools
}

\begin{abstract}
:
Even with the range of information available on the harm caused by continuous exposure to nicotine, tobacco is still in the second position in the ranking of legal drugs most consumed according to the National Cancer Institute (INCA). This research is part of a larger project whose central theme is the implementation of educational activities on smoking and adolescence, in schools in the city of Londrina - PR, focusing on adolescents aged between 14 and 20 years. The objectives of the study were to identify the cause of young people's interest in smoking, as well as the interest of adolescent smokers in the consumption of different forms of smoking (cigarette, hookah and/or electronic cigarette/vape) and what are the influences for consumption smoking among adolescents. A questionnaire was applied to high school students, enrolled in public schools in the peripheral and central region of the city of Londrina - PR. The results indicated that $39.4 \%$ were already interested and had already tried one of the three forms of smoking (cigarette, hookah and/or electronic cigarette/vape), in addition, the hookah smoking habit was the one that most appeared among students. It is noteworthy that the school, due to its characteristics, becomes an excellent environment for carrying out educational practices to prevent cigarette consumption among adolescents.
\end{abstract}

Keywords: Smoking, adolescence, school, health education.

\section{Hábito de fumar entre adolescentes de escuelas brasileñas}

\section{Resumen:}

Incluso con el abanico de información disponible sobre los daños causados por la exposición continua a la nicotina, el tabaco aún ocupa el segundo lugar en el ranking de drogas legales más consumidas según el Instituto Nacional del Cáncer (INCA). Esta investigación es parte de un proyecto más amplio cuyo tema central es la implementación de actividades educativas sobre tabaquismo y adolescencia, en escuelas de la ciudad de Londrina - PR, con foco en adolescentes de entre 14 y 20 años. Los objetivos del trabajo fueron identificar la causa del interés de los jóvenes por el hábito de fumar, así como el interés del fumador adolescente por el consumo de diferentes formas de fumar (cigarrillo, narguile y/o cigarrillo electrónico/vape) y cuáles son las influencias del consumo de tabaco entre los adolescentes. Se aplicó un cuestionario a estudiantes de secundaria, matriculados en escuelas públicas de la región periférica y central de la ciudad de Londrina - PR. Los resultados indicaron que el 39,4\% ya estaba interesado y ya había probado alguna de las tres formas de fumar (cigarrillo, narguile y/o cigarrillo electrónico/vape), además, el hábito de fumar narguile fue el que más apareció entre los estudiantes. Es de destacar que la escuela, por sus características, se convierte en un excelente entorno para la realización de prácticas educativas para prevenir el consumo de cigarrillos entre los adolescentes.

Palabras clave: Tabaquismo, adolescencia, escuela, educación para la salud.

\section{INTRODUÇÃo}

No início da adolescência é observado um latente período de transformações morfológicas, fisiológicas e de novos comportamentos. Tal período discorre em situações suscetíveis à aquisição de práticas que podem prejudicar a saúde, como sedentarismo, tabagismo e consumo de substâncias que configuram os alicerces inerentes a comportamentos de risco (INCA, 2007). Segundo Turner, Mermelstein e Flay (2004), quando 
exposto ao tabagismo, o adolescente produz ao seu convívio uma gama de fatores que impactam diretamente no seu bem-estar, dentre eles, por estarem em posse de substâncias proibidas a menores de 18 anos, necessitam por muitas vezes de aporte financeiro para sustentar seu vício, prejudicando, consequentemente, a saúde de seus familiares não fumantes com fumo passivo e, em algumas ocasiões, induzindo outros familiares a consumir tal substância, como irmãos e parentes mais jovens.

Cerca de 1,8 milhão de adolescentes já usou pelo menos algum produto derivado de tabaco, sendo esses jovens da faixa etária de 12 a 17 anos (MACIEL, 2016). Dessa forma, Ferreira et al. (2012) reiteram o papel da escola na formação de pessoas críticas e construtores de conhecimento, visando preparar e educar o aluno para os cuidados com sua saúde; sendo assim, propostas pedagógicas que tratem dessa temática são fundamentais para a educação da saúde dos adolescentes. No entanto, consideramos relevante conhecer os hábitos de consumo do tabaco entre os adolescentes para preparar práticas pedagógicas que possam ser mais efetivas, de modo a conscientizá-los sobre os malefícios do cigarro.

O tabagismo é reconhecido como uma comorbidade crônica, causando doenças crônico-degenerativas pela dependência da substância nicotina, presente em produtos à base de tabaco e similares (WORSNOP, 2003; OMS, 2003). De acordo com o INCA (2007), o tabaco encontra-se na segunda posição no ranking de drogas mais experimentadas pelos jovens.

Em relação a isso, segundo Menezes et al. (2014), os indivíduos se contrapõem entre a fase infantil, livre de obrigações e deveres, e a adolescência propriamente dita, que é a fase de transição entre a infância e a vida adulta. Os adolescentes vivenciam mudanças sociais e psicológicas, o que os torna vulneráveis e influenciáveis no meio a que pertencem; isso faz com que adotem práticas maléficas, como o uso de drogas lícitas e ilícitas, principalmente tabagismo e consumo de bebidas alcoólica (SAITO, 2000; AYRES et al., 2003).

De acordo com Schneider e Alves,

O uso dessas substâncias pelos pais e pelos amigos pode motivar sua experimentação pelo adolescente. No entanto, além da vulnerabilidade individual dos jovens, eles também estão expostos à vulnerabilidade institucional, em função da escassez de ações voltadas diretamente para essa faixa etária e da carência de profissionais especializados para o atendimento de adolescentes. Nesse contexto, é fundamental o desenvolvimento de ações de promoção da saúde que sensibilizem os jovens e os orientem sobre os malefícios do cigarro, fazendo-se necessário que o Estado 
desenvolva políticas públicas que protejam a saúde de adolescentes em relação ao uso do tabaco e outras drogas (SCHNEIDER; ALVES, 2009, p. 1060-1061).

O narguilé é outra forma de utilização do tabaco que se tornou uma prática sociável, o que corrobora com misticismo de ser uma prática não prejudicial; adolescentes e jovens adultos estão dentre os seus maiores entusiastas. Tratando-se de um tipo de consumo de droga lícita, é colocado como precursor para a aderência do uso de outras formas de tabaco (GROSS, 2016).

Por outro lado, o uso de narguilé difunde-se no paradigma errado quanto ao grau de perigo, ou seja, na cultura popular se diz que o uso ou consumo de narguilé não afetará a saúde como o cigarro tradicional. Porém, de acordo com pesquisas e estudos recentes, comprova-se que o uso prolongado de narguilé pode causar câncer de boca, de pulmão, de bexiga e doenças respiratórias (INCA, 2017).

Apesar das informações sobre os riscos do uso contínuo de tabaco estejam sempre em evidência, o conhecimento sobre as implicações causadas por esse hábito tanto para fumantes quanto para fumantes passivos ainda não é de total consciência dessa classe. Estima-se que, no Brasil, o vício pela nicotina tem como causas principais do câncer, doença cardíaca e doença pulmonar obstrutiva crônica (DPOC). Tendo em vista a ineficiência das propostas de intervenções, o número anual de mortes é crescente, entretanto, se o investimento em saúde, em profissionais qualificados e em propostas de intervenção fossem eficazes, as mortes poderiam ser evitadas (PINTO et al., 2019).

A exposição precoce ao tabagismo acarreta inúmeras complicações para a saúde dos adolescentes e pode ocorrer em curto, médio e longo prazo. Jovens fumantes consomem 3 vezes mais bebidas alcoólicas comparados aos não fumantes (WHO, 2008); além disso, o uso contínuo do tabaco reflete em consequências para a vida adulta. Segundo Peto et al. (2000), indivíduos que adquirem o hábito de fumar mais cedo são mais propensos ao desenvolvimento de câncer de pulmão, além de câncer em outros locais, como trato intestinal, renal, cavidade oral entre outros. Além disso, o tabaco é responsável pela incidência de doenças cardiovasculares e acidentes vasculares cerebrais (ONUBR, 2018).

Estudos feitos no Brasil demonstram que há grande prevalência de adolescentes fumantes. Os números variam entre $1 \%$ a $37 \%$, e tais resultados acentuam a necessidade de subsídios e políticas preventivas para o controle do tabagismo. Além disso, campanhas 
antitabagistas e criação de leis para impedir o acesso ao tabaco aos menores de 18 anos vêm sendo colocadas em prática pelo Ministério da Saúde. O Sistema Único de Saúde (SUS) também vem buscando soluções que possibilitem aos dependentes do tabaco o tratamento de forma gratuita (NOGUEIRA; FUMO; SILVA, 2004).

Esta pesquisa teve início com um levantamento de dados por meio da aplicação de um questionário, no qual se utilizou como base um questionário-piloto (adaptação do estudo de Pinto et al., 2013), com o objetivo de identificar a causa e influência do interesse dos jovens pelo hábito do tabagismo e identificar também o interesse do adolescente fumante pelo consumo das diferentes formas do tabagismo (cigarro, narguilé e/ou cigarro eletrônico/vape).

\section{METODOLOGIA}

Esta pesquisa tem uma abordagem qualitativa e usa como instrumento a produção de um questionário; tem como objetivo levantar dados sobre os hábitos relacionados ao tabagismo entre os adolescentes do ensino médio de escolas públicas do município de Londrina/PR.

O público-alvo selecionado para participar foram adolescentes com idade entre $14 \mathrm{e}$ 20 anos, devidamente matriculados em alguma das três séries do Ensino Médio das seis escolas selecionadas, sendo estas localizadas na região central e periférica do município de Londrina/PR. A escolha das escolas se deu a partir da participação destas nos projetos vinculados à Universidade Estadual de Londrina: Programa Institucional de Bolsa de Iniciação à Docência (PIBID) e Residência Pedagógica, que são programas de iniciação à docência (CAPES/MEC).

Com relação à obtenção dos dados, elaborou-se um questionário em cuja construção utilizou-se como base artigos relacionados com o tema do tabagismo e adolescência, como: Oliveira (2009), Amaral (2010), Vicente (2010); Barreto et al. (2014), Malta et al. (2018), Passos e Oliveira (2019) e um questionário piloto adaptado de Pinto et al. (2013). 
O questionário foi formado por 21 questões, sendo duas discursivas e 19 objetivas (Apêndice I). $\mathrm{O}$ instrumento trata de questões gerais e questões relacionadas ao cigarro, narguilé e cigarro eletrônico, sendo validado por professores da área da educação e da saúde. Por fim, o questionário foi inserido na plataforma "Formulários Google", onde foi gerado um link de acesso aos alunos para a obtenção das respostas.

Para organização dos dados obtidos no levantamento, foi utilizada a planilha eletrônica Microsoft Excel e, para avaliar o perfil dos estudantes de escolas vinculadas ao Projeto, foi realizado o teste de aderência qui-quadrado (AGRESTI, 2007) para testar as seguintes hipóteses: Hipótese nula (as porcentagens observadas e esperadas são iguais) contra Hipótese alternativa (existe pelo menos uma diferença entre as porcentagens observadas e esperadas). Fixando o nível de significância em $5 \%$, o critério de rejeição da hipótese nula é definido como $p$-valor menor do que $5 \%$. Além disso, para ajudar na tomada de decisão sobre a rejeição da hipótese nula, também foi utilizado o teste G2 (MCDONALD, 2014), que é uma alternativa ao teste qui-quadrado (AGRESTI, 2007). Para auxiliar na análise de dados, foi empregado o software estatístico R versão 4.0.3 (R CORE TEAM, 2021).

\section{RESULTADOS}

Participaram da pesquisa 154 pessoas do sexo feminino (65\%) e 83 do sexo masculino (35\%), totalizando 264 participantes. A idade dos estudantes participantes da pesquisa variou de 14 a 20 anos, sendo em sua maioria respondentes de 16 e 17 anos, seguidos pelos de 15 e 18 anos.

Na Tabela 1, foram avaliados parâmetros em relação ao interesse dos adolescentes em fumar cigarro, narguilé e cigarro eletrônico/vape, respectivamente. Os resultados da Tabela 1 levam à rejeição da hipótese nula ( $p$-valor $=0,104)$. Logo, temos indicativos de que a porcentagem de alunos interessados em tabaco não difere da porcentagem de alunos que nunca tiveram o interesse. 
Tabela 1 - Teste de aderência dos dados de interesse em fumar cigarro, narguilé ou cigarro eletrônico/vape

\begin{tabular}{ccccc}
\hline & & & \multicolumn{2}{c}{$p$-valor } \\
\cline { 4 - 5 } Categorias & Total & Porcentagem (\%) & Qui-quadrado & Gtest \\
\hline Fumar tabaco & & & 0,104 & 0,104 \\
Interessados & 131 & 55 & & \\
Nunca teve interesse & 106 & 45 & & \\
\hline
\end{tabular}

Fonte: Da pesquisa.

Foi observado a partir dos dados que pode haver uma diferença entre homens e mulheres quanto ao interesse em fumar algum tipo de tabaco. Além disso, na categoria interessados, as idades de maior frequência foi 16 e 17 anos, com destaque para a idade de 17 no grupo feminino. Por outro lado, na categoria nunca teve interesse, as idades foram 16 e 17 anos. Foram feitas análises de acordo com as categorias de interesse em fumar cigarro, narguilé ou cigarro eletrônico/vape, conforme descrito a seguir.

\section{a) Nunca teve interesse}

Tabela 2 - Teste de aderência para os dados alunos que nunca tiveram interesses em fumar

\begin{tabular}{|c|c|c|c|c|}
\hline \multirow[b]{2}{*}{ Variável } & \multirow[b]{2}{*}{ Total } & \multirow[b]{2}{*}{ Porcentagem (\%) } & \multicolumn{2}{|l|}{$p$-valor } \\
\hline & & & Qui-quadrado & Gtest \\
\hline Sexo & & & 0,012 & 0,011 \\
\hline Feminino & 66 & 62 & & \\
\hline Masculino & 40 & 38 & & \\
\hline Idade & & & $<0,001$ & $<0,001$ \\
\hline 14 a 15 anos & 31 & 29 & & \\
\hline 16 a 17 anos & 59 & 56 & & \\
\hline 18 ou mais & 13 & 12 & & \\
\hline Branco & 3 & 3 & & \\
\hline Questão 1* & & & $<0,001$ & $<0,001$ \\
\hline Não & 33 & 31 & & \\
\hline Sim & 73 & 69 & & \\
\hline Questão 2** & & & $<0,001$ & $<0,001$ \\
\hline Não & 30 & 28 & & \\
\hline Sim & 76 & 72 & & \\
\hline
\end{tabular}

*Questão 1: Você tem conhecimento que o narguiléé uma forma diferenciada do uso do tabaco?

**Questão 2: Você já teve alguma informação sobre o uso do tabaco? (Palestras em escolas, comerciais na televisão, mídia etc.).

Fonte: da pesquisa. 
Por meio da Tabela 2, é possível observar que a porcentagem de mulheres (62\%) é maior do que a porcentagem de homens em relação a nunca ter tido interesse de fumar ( $p$ valor $=0,012 / 0,011$ ). Também rejeitamos a hipótese nula ( $p$-valor $<0,001$ ), isto é, entre os alunos que nunca tiveram interesse em fumar, a faixa de idade é de 16 e 17 anos (56\%), seguido dos alunos de 14 e 15 anos (29\%); os que nunca tiveram interesse fumar sabem que o narguilé é uma forma diferenciada de uso do tabaco (69\%), e os estudantes que nunca tiveram interesse fumar já tiveram alguma informação sobre o uso do tabaco (72\%).

\section{b) Interessados em fumar}

Tabela 3 - Teste de aderência para os dados de alunos interessados em fumar

\begin{tabular}{|c|c|c|c|c|c|}
\hline \multirow{2}{*}{\multicolumn{2}{|c|}{ Variável }} & \multirow[b]{2}{*}{ Total } & \multirow[b]{2}{*}{ Porcentagem (\%) } & \multicolumn{2}{|l|}{$p$-valor } \\
\hline & & & & Qui-quadrado & Gtest \\
\hline Sexo & & & & $<0,001$ & $<0,001$ \\
\hline & Feminino & 88 & 68 & & \\
\hline & Masculino & 43 & 33 & & \\
\hline Idade & & & & $<0,001$ & 0,001 \\
\hline & 14 a 15 anos & 17 & 13 & & \\
\hline & 16 a 17 anos & 87 & 66 & & \\
\hline & 18 ou mais & 27 & 21 & & \\
\hline Questão 1 & & & & $<0,001$ & $<0,001$ \\
\hline & Não & 11 & 8 & & \\
\hline & Sim & 120 & 92 & & \\
\hline Questão 2 & & & & $<0,001$ & $<0,001$ \\
\hline & Não & 16 & 12 & & \\
\hline & Sim & 99 & 76 & & \\
\hline & Branco & 16 & 12 & & \\
\hline
\end{tabular}

*Questão 1: Você tem conhecimento que o narguilé é uma forma diferenciada do uso do tabaco?

**Questão 2: Você já teve alguma informação sobre o uso do tabaco? (Palestras em escolas, comerciais na televisão, mídia etc.).

Fonte: da pesquisa.

Por meio da Tabela 3, foi possível verificar que a porcentagem de mulheres (68\%) é maior do que a porcentagem de homens em relação a interessados em fumar. Entre os alunos interessados em fumar, a faixa de idade é de 16 e 17 anos (66\%), seguido dos estudantes de 18 ou mais (21\%). Os alunos interessados em fumar já tiveram alguma informação sobre o uso do tabaco $(76 \%)$ ( $p$-valor $<0,001)$. 
O próximo passo foi considerar apenas a categoria de alunos interessados em fumar cigarro, narguilé ou cigarro eletrônico/vape. Foi-lhes perguntado se fumaram algum tipo de tabaco, e o resultado demonstrou o hábito preferencial pelo narguilé, seguido do cigarro eletrônico/vape e por último o cigarro. Por meio da Tabela 4, tivemos as evidências que entre os alunos que fumaram mais de uma vez a preferência é pelo narguilé $(68 \%)$ ( $p$-valor $<0,001)$. Entre os alunos que fumaram apenas uma vez, a tendência é que prefiram o cigarro eletrônico/vape. Porém, não é possível afirmar que a porcentagem dos alunos que escolheram cigarro eletrônico/vape seja estatisticamente diferente das demais porcentagens, ao nível de significância de $5 \%$.

Tabela 4 - Teste de aderência para os dados dos alunos que responderam SIM para a questão você já fumou cigarro, narguilé ou cigarro eletrônico/vape?

\begin{tabular}{|c|c|c|c|c|}
\hline \multirow[b]{2}{*}{ Tabaco } & \multirow[b]{2}{*}{ Total } & \multirow[b]{2}{*}{ Porcentagem (\%) } & \multicolumn{2}{|c|}{$p$-valor } \\
\hline & & & Qui-quadrado & Gtest \\
\hline Mais de uma vez & & & $<0,001$ & $<0,001$ \\
\hline Cigarro & 11 & 10 & & \\
\hline Narguilé & 79 & 68 & & \\
\hline Cigarro eletrônico/vape & 26 & 22 & & \\
\hline Uma vez & & & 0,054 & 0,058 \\
\hline Cigarro & 49 & 31 & & \\
\hline Narguilé & 42 & 27 & & \\
\hline Cigarro eletrônico/vape & 66 & 42 & & \\
\hline
\end{tabular}

Fonte: da pesquisa.

Logo, sobre os alunos que fumaram mais de uma vez narguilé, temos que a faixa etária predominante entre os alunos é de 11 a 15 anos (76\%) com a frequência semanal de 1 a 5 vezes (82\%) ( $p$-valor $<0,001)$. Rejeitamos hipótese nula ( $p$-valor $<0,001$ ), portanto temos evidências de que a faixa etária predominante entre os alunos que fumaram apenas uma vez narguilé é, também, de 11 a 15 anos (60\%).

$\mathrm{Na}$ tabela 5, apresentamos as repostas referentes à frequência no uso do cigarro, narguilé ou cigarro eletrônico/vape consumido por semana. 
Tabela 5 - Frequência do uso de cigarro, narguilé ou cigarro eletrônico/vape durante a semana.

\begin{tabular}{|c|c|c|c|c|c|c|}
\hline \multirow[t]{2}{*}{ Frequência } & \multicolumn{2}{|c|}{ Cigarro } & \multicolumn{2}{|c|}{ Narguilé } & \multicolumn{2}{|c|}{$\begin{array}{c}\text { Cigarro } \\
\text { Eletrônico/vape }\end{array}$} \\
\hline & $\mathbf{n}$ & $\%$ & $\mathbf{N}$ & $\%$ & $\mathbf{n}$ & $\%$ \\
\hline 1 a 5 vezes na semana & 6 & 2,3 & 67 & 25,4 & 18 & 6,8 \\
\hline 6 a 10 vezes na semana & 0 & 0 & 4 & 1,5 & 4 & 1,5 \\
\hline $\begin{array}{l}11 \text { ou mais vezes na } \\
\text { semana }\end{array}$ & 3 & 1,1 & 10 & 3,8 & 4 & 1,5 \\
\hline Nenhuma vez na semana & 255 & 96,6 & 181 & 68,6 & 238 & 90,2 \\
\hline Em branco & 0 & 0 & 2 & 0,8 & 0 & 0 \\
\hline Total & 264 & $100 \%$ & 264 & $100 \%$ & 264 & $100 \%$ \\
\hline
\end{tabular}

Fonte: Da pesquisa.

Em relação à frequência do uso de uma das três formas de tabagismo durante a semana, nota-se que a mais significativa diz respeito à quantidade de uma a cinco vezes na semana, sendo $25,4 \%$ no uso do narguilé, seguido do cigarro eletrônico/vape com 6,8\% e, por último, o cigarro, com uma porcentagem de $2,3 \%$.

É perceptível a preferência dos adolescentes pelo uso do narguilé, seguido do cigarro eletrônico/vape, isso porque seu uso pode ser considerado uma ação precursora para a iniciação do fumo de cigarros. Para ilustrar isso, elaborou-se um gráfico referente às principais influências para o início do tabagismo (Figura 1). 
Figura 1. Frequências das respostas dos alunos que são ou foram usuários de tabaco, sobre a questão "Qual a principal influência para você ter começado a fumar"

\section{Principal influência}

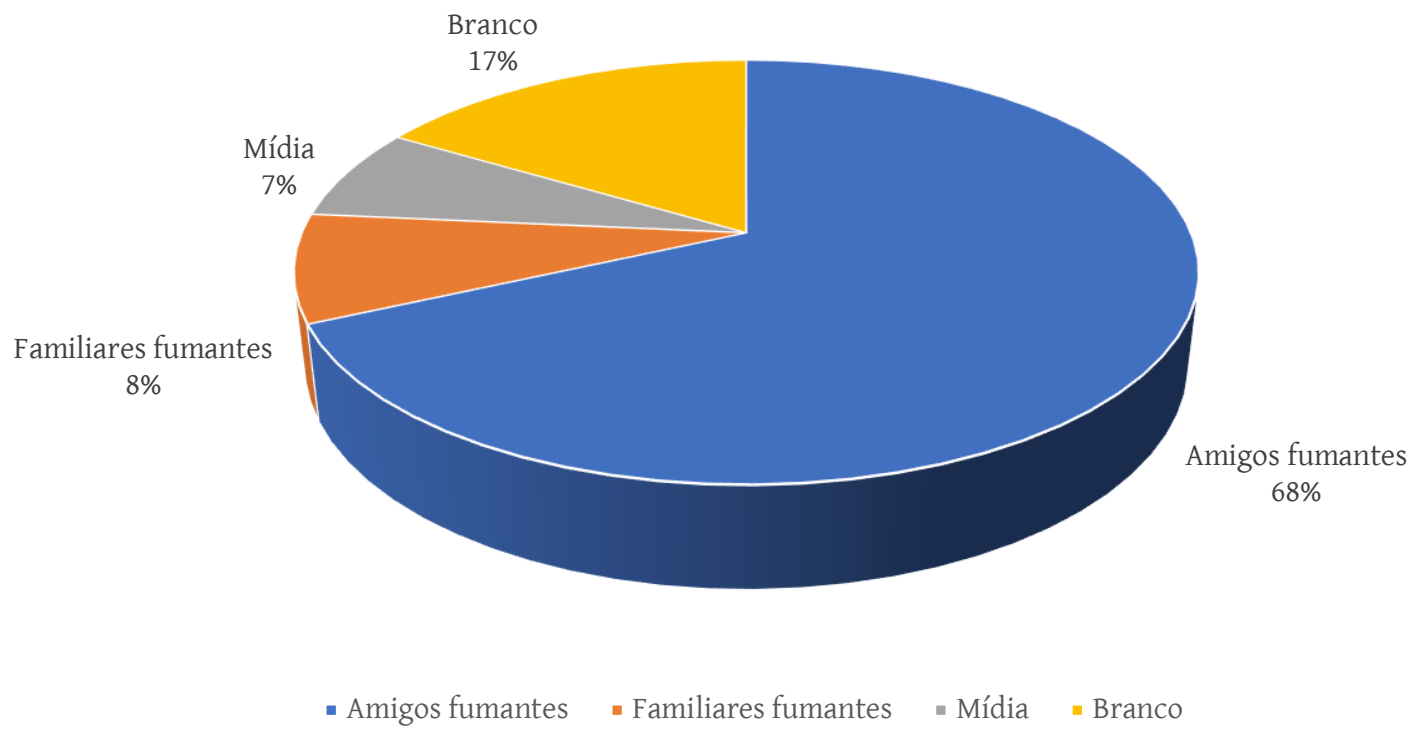

Fonte: Da pesquisa.

Observa-se na Figura 1 que a influência para a experimentação ou o início de fumar está relacionado com amigos (68\%) e familiares fumantes (8\%), seguido pela influência da mídia (7\%).

A partir da Tabela 6, rejeitamos a hipótese nula (p-valor<0,001), ou seja, temos evidências que os alunos que fumaram mais de uma vez cigarro, narguilé ou cigarro eletrônico/vape tiveram como principal influência os amigos (68\%).

Tabela 6 - Teste de aderência para os dados de alunos que fumaram mais de uma vez cigarro, narguilé ou cigarro eletrônico/vape

\begin{tabular}{|c|c|c|c|c|}
\hline \multirow[b]{2}{*}{ Variável } & \multirow[b]{2}{*}{ Total } & \multirow[b]{2}{*}{ Porcentagem (\%) } & \multicolumn{2}{|c|}{$p$-valor } \\
\hline & & & Qui-quadrado & Gtest \\
\hline Influência & & & $<0,001$ & $<0,001$ \\
\hline Amigos fumantes & 58 & 68 & & \\
\hline Familiares fumantes & 7 & 8 & & \\
\hline Mídia & 6 & 7 & & \\
\hline Branco & 14 & 17 & & \\
\hline
\end{tabular}

Fonte: da pesquisa. 
A Figura 2 diz respeito a situações em que o tabagismo está associado ao cotidiano desses indivíduos para que haja o entendimento da relação do hábito de fumar com o momento em que esses jovens estão vivenciando, seja ele uma ansiedade, alegria, entre outros, isto é, associado a momentos de prazer ou de tristezas.

Figura 2. Frequência das respostas dos alunos participantes da pesquisa, sobre a questão "A que situações o tabagismo está associado no seu dia a dia".

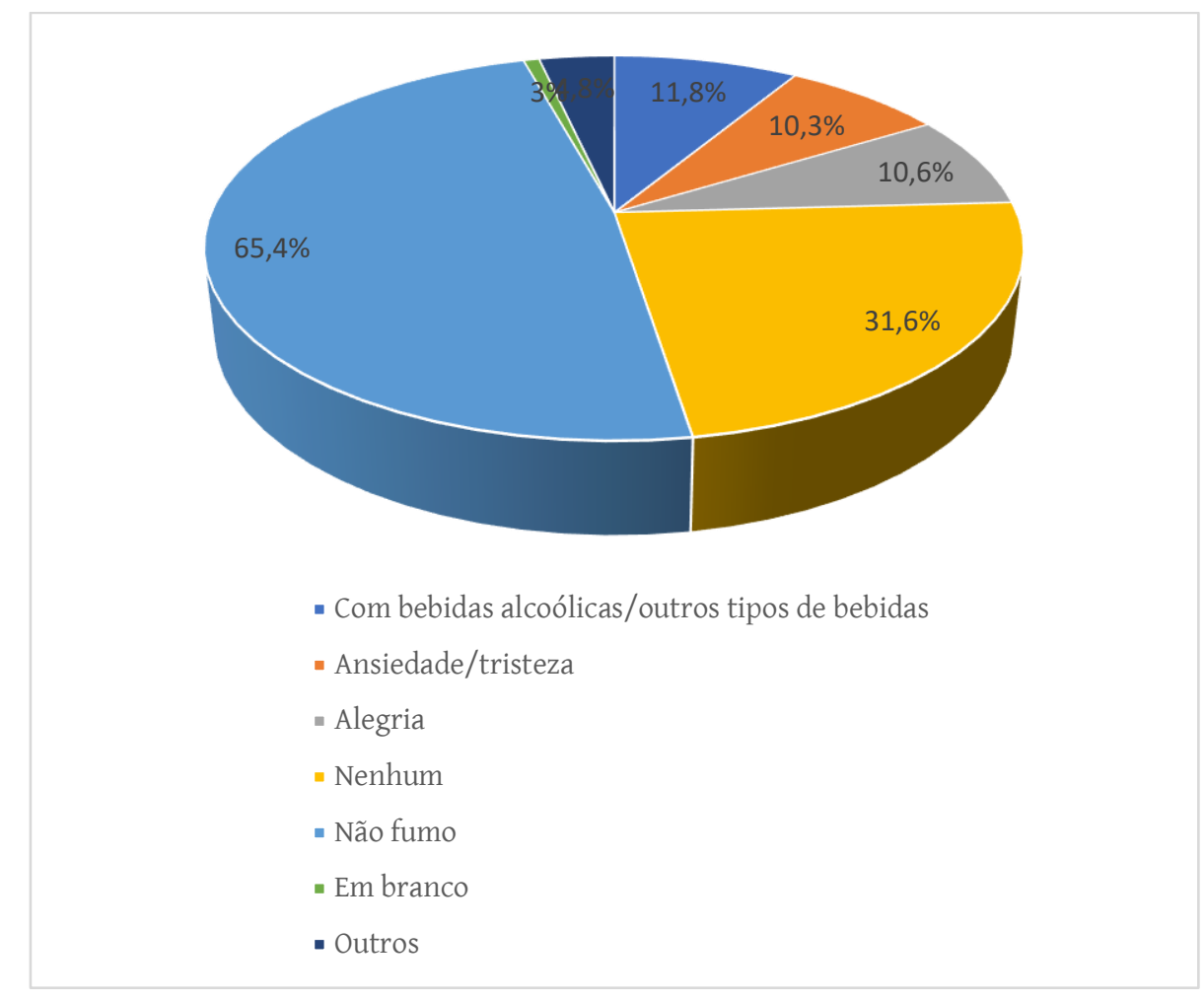

Fonte: Da pesquisa.

Na presente pesquisa, é possível observar (Figura 2) que uma parte dos respondentes não fuma (65,4\%); contudo, também há associação do tabaco com bebidas alcoólicas e outros tipos de bebidas (11,8\%). Além disso, foi demonstrado que há associação nas situações que trazem alegria para o indivíduo (10,6\%), que culmina na utilização de tabaco. Já ansiedade e tristeza (10,3\%) estão relacionadas com fuga de emoções e subterfúgios de possíveis circunstâncias desagradáveis. Na opção nenhuma relação, 31,6\% dos respondentes se identificaram com ela. De acordo com a pesquisa, o resultado denominado outros $(4,8 \%)$ vai desde simplesmente a vontade de fumar até um ato naturalmente executado após a prática 
sexual, que configuram um leque variado de comportamentos correlacionados com o hábito de fumar. Vislumbra-se no gráfico que algumas pessoas tendem a assimilar tais situações indiretamente com o uso do tabaco.

Dentre os participantes, $81,4 \%$ alegaram que têm conhecimento que o narguilé é uma forma diferenciada do uso do tabaco; os demais 18,6\% desconheciam tal informação. Enquanto, em relação ao acesso às informações por meio de palestras, mídias, comerciais de televisão e outros meios sobre o uso de tabaco e seus malefícios, 79,2 \% dos respondentes afirmaram já ter tido acesso e outros 20,8 \% alegaram que não foram alcançados por tais informações.

Quanto às influências para o consumo, 28,8\% fuma por vontade própria, seguido de 6,4\% fumam por conta da observação de amigos fumando e, por último, 3\% são influenciados por familiares fumantes. A utilização de bebidas alcoólicas é relatada por $10 \%$ dos respondentes como uma influência para a vontade de fumar.

Sobre o questionamento se houve algum sintoma como tosse, falta de ar, entre outros quando da utilização do tabaco, obtivemos os seguintes resultados: 13,8 \% alegaram sim e $86,2 \%$ não.

\section{DISCUSSÃo}

A principal influência para o interesse e para uma primeira experimentação está relacionada à fase da adolescência, em que os jovens buscam autonomia e independência, $e$ deixam-se levar pelo marketing produzido pela indústria do tabaco, que traz imagens de sucesso e glamour associado ao tabagismo (NASCIMENTO et al., 2005). É perceptível a preferência dos adolescentes pelo uso do narguilé, seguido do cigarro eletrônico/vape, isso porque seu uso pode ser considerado uma ação precursora para a iniciação do fumo de cigarros (MAKIAK; WARD; EISSENBERG, 2007). 0 narguilé está associado a uma crença de que é menos prejudicial que outras formas de tabagismo; além disso, é de fácil acesso. Entretanto, é sabido que possui uma quantidade elevada de nicotina, o que o torna prejudicial à saúde, assim como o tabaco, causando doenças cardiovasculares e respiratórias, além de câncer no 
pulmão e em diversos outros locais do organismo humano (REVELES; SEGRI; BOTELHO, 2013). Além disso, como já foi mencionado, os malefícios do tabaco também estão presentes no uso do narguilé e do cigarro eletrônico/vape, devido à fumaça conter as mesmas substâncias presentes no tabaco, tais como: nicotina, monóxido de carbono, hidrocarbonetos e toxinas (MAKIAK, 2011).

Quanto à média de idade de iniciação do uso de cigarro, narguilé e cigarro eletrônico/vape, a literatura aponta que uma parcela significativa de adolescentes tem um início precoce no vício de fumar, o que acontece entre 13 e 15 anos (MALCON; MENEZES; CHATKIN, 2003). Um outro fator importante que facilita o início da experimentação está interligado aos conflitos familiares, bem como às influências de meios sociais em que vivem (PASQUALOTTI et al., 2006). Na adolescência, o número de fumantes é elevado devido à idade de início ser cada vez mais precoce, enquanto a prevalência entre os adultos tende a diminuir com o passar dos anos (FLAY, 1992).

A influência para a experimentação ou o início de fumar está relacionado com amigos e familiares fumantes, pois, por ver seus pais ou irmãos mais velhos fumando e por conviver diariamente com eles, colocando-o na condição de fumante passivo, inconscientemente adere ao ato prejudicial à sua saúde. Um outro ponto que vale salientar são as relações sociais, já que amigos e colegas exercem certa influência nos indivíduos não fumantes; isso se dá por conta do processo de aceitação de determinados grupos sociais, nos quais se espelham e cujos comportamentos reproduzem (MALCON; MENEZES; CHATKIN, 2003).

Na presente pesquisa, é possível observar que uma parte dos respondentes não fuma, contudo, a associação do tabaco com bebidas alcoólicas e outros tipos de bebidas faz com que o indivíduo passe a ter uma falsa segurança, sentindo-se mais corajoso para enfrentar dificuldades com a ingestão de substâncias alcoólicas (VIEIRA et al., 2008). Foi demonstrado que há associação do uso do tabaco nas situações que trazem alegria, ansiedade e tristeza.

Dentre os participantes, alguns alegaram que têm conhecimento que o narguilé é uma forma diferenciada do uso do tabaco e os demais desconheciam tal informação. Por muito tempo, o uso do narguilé foi visto como menos prejudicial à saúde, em relação à prática de fumar cigarro. Porém, os estudos científicos evidenciam os seus malefícios, visto que a combustão do fumo é incompleta, por isso há maiores liberações de produtos tóxicos 
(SHIHADEH et al., 2015). Em relação às substâncias presentes na fumaça do narguilé, estudos comprovam que, dentre 300 substâncias identificadas, 23 são consideradas cancerígenas (HAMMAL, 2015).

De acordo com Poletto et al. (1991) e Malcon, Menezes e Chatkin (2003), grande parte dos jovens relatou que deu início à prática de fumar por conta da observação constante de amigos fumando. Além disso, a maioria relatou que fuma por vontade própria, e uma minoria fuma por causa da influência de familiares. O uso de bebidas alcoólicas é relatado como uma influência para a vontade de fumar, segundo o levantamento do Centro Brasileiro de Informação Psicotrópica (CEBBRID), álcool e tabaco são as drogas que adolescentes e jovens começam a usar mais cedo.

Conforme Santos et al. (2011), as enfermidades adquiridas devido à prática do uso do tabaco acarretarão eminentes riscos pulmonares, como tosse frequente e seca, também falta de ar, problemas cardiovasculares e câncer; o uso prolongado do tabaco, por sua vez, refletirá nas capacidades cognitivas, consequentemente levando ao déficit de memória.

A disseminação das informações sobre os malefícios do consumo de tabaco a partir de palestras, mídias, comerciais de televisão e outros meios, colabora a cessação ainda que parcial de fumantes de longo prazo e de iniciantes; além desses meios citados, há também uma grande mobilização em ações educativas através de campanhas de conscientização e melhorias a respeito de implantações de programas de prevenção tanto no ambiente de trabalho quanto em áreas de ambientes comuns, ou seja, ambientes de convivência de pessoas fumantes ou não (CAVALCANTE, 2005).

\section{CONSIDERAÇÕES FINAIS}

Os resultados obtidos nesta pesquisa nos permitem concluir que o início da experimentação em alguma das três formas de tabagismo se dá entre os 13 e 15 anos, com destaque para o uso do narguilé, o qual foi o que obteve maior número de respostas e maior frequência no consumo durante a semana, mostrando-se altamente significativo dentro dos 
resultados, visto ser popularmente disseminado entre os jovens (MALCON; MENEZES; CHATKIN, 2003). A principal influência observada no estudo para a primeira experimentação está relacionada aos amigos fumantes, seguida de familiares fumantes, enquanto as situações que mais estão associadas ao tabagismo são o consumo de bebidas alcoólicas e outros tipos de bebidas.

A grande influência do interesse ao tabagismo está relacionada à fase da adolescência, em que os jovens buscam autonomia e independência, e deixam se levar pelo marketing produzido pela indústria do tabaco. O narguilé está associado a uma crença de que é menos prejudicial que outras formas de tabagismo. Este, ao contrário do que os jovens acreditam, possui uma elevada quantidade de nicotina, evidenciando que os malefícios do tabaco também estão presentes no uso do narguilé, causando doenças cardiovasculares e respiratórias, além de câncer no pulmão e outros (REVELES; SEGRI; BOTELHO, 2013).

Visto que o tabagismo tem se tornado um hábito frequente, estudos apontam que 23 das 300 substâncias identificadas na fumaça do narguilé são consideradas cancerígenas (HAMMAL, 2015). Outro fator de grande importância para o início da prática são os conflitos familiares, bem como influências dos meios sociais e familiares fumantes, facilitando o início da experimentação (PASQUALOTTI et al., 2006).

Tendo em vista o alto índice de jovens fumantes, é de extrema necessidade subsídios e políticas preventivas para o controle do tabagismo, além de campanhas antitabagistas e criação de leis para impedir o acesso ao tabaco por menores de 18 anos, o que vem sendo colocado em prática pelo Ministério da Saúde. O Sistema Único de Saúde (SUS) também vem buscando soluções que possibilitam aos dependentes do tabaco o tratamento de forma gratuita (NOGUEIRA; FUMO; SILVA, 2004). Entretanto, a escola também possui papel essencial na prevenção ao hábito do tabagismo, visto que é um ambiente de apoio que compreende a formação acadêmica e parte da construção social e ética do jovem brasileiro, podendo promover ações para a saúde e enfatizar os bons hábitos. 


\section{REFERÊNCIAS BIBLIOGRÁFICAS}

AGRESTI, A. An introduction to categorical data analysis. 2. ed. New York: John Wiley \& Sons, 2007.

AMARAL, V. G. A adolescência e o tabaco. Caracterização do Consumo Numa População Escolar. Dissertação (Mestrado em Medicina) - Universidade Beira do Interior, Covilhã, Portugal, 2010. Disponível em: <https://ubibliorum.ubi.pt/bitstream/10400.6/739/1/Mestrado\%20Vanessa\%20Goulart\%20Amaral.pdf>.

Acesso em: 28 maio 2020.

AYRES, J. R. C. M.; FRANÇA JÚNIOR, I.; CALAZANS, G. J.; SALETTI FILHO, H. C. O conceito de vulnerabilidade e as práticas de saúde: novas perspectivas e desafios. In: CZERESNIA, Dina; FREITAS, Carlos Machado de. Promoção da saúde: conceitos, reflexões, tendências, v. 2. Rio de Janeiro: Fiocruz, 2003. p. 121-144.

BARRETO, S. M.; GIATTI, L.; OLIVEIRA-CAMPOS, M.; ANDREAZZI, M. A.; MALTA, D. C. Experimentação e uso atual de cigarro e outros produtos do tabaco entre escolares nas capitais brasileiras (PeNSE 2012). Revista Brasileira de Epidemiologia, v. 17, p. 62-76, São Paulo, 2014. Disponível em: <https://www.scielo.br/pdf/rbepid/v17s1/pt_1415-790X-rbepid-17-s1-00062.pdf>. Acesso em: 28 maio 2020.

CAVAlCANTE, T. M. O controle do tabagismo no Brasil: avanços e desafios. Revista de Psiquiatria Clínica, v. 32, n. 5, p. 283-300, São Paulo, 2005. Disponível em: <https://www.scielo.br/pdf/rpc/v32n5/27703.pdf>. Acesso em: 28 maio 2020 .

FERREIRA, I. do R. C.; VOSGERAU, D. S. R.; MOYSÉS, S. J.; MOYSÉS, S. T. Diplomas Normativos do Programa Saúde na Escola: análise de conteúdo associada à ferramenta ATLAS TI. Ciência \& Saúde Coletiva, v. 17, n. 12, p. 33853398, Rio de Janeiro, 2012. Disponível em: <https://www.scielo.br/pdf/csc/v17n12/23.pdf>. Acesso em: 10 jul. 2020.

FLAY, B. R. Youth tobacco use: risk, patterns and control. In: SLADE, J.; ORLEANS, C. T. (ed.) Nicotine Addiction: Principles and Management. London: Oxford, 1992. p. 129-142. Disponível em: <http://pepsic.bvspsi.org.br/pdf/rip/v40n2/v40n2a09.pdf>. Acesso em: 28 maio 2020.

GROSS, J. L. Os perigos do uso do narguilé. São Paulo: A. C. Camargo Câncer Center. 2016. Disponível em: <http://www.jorbras.com.br/portal/index.php?option=com_content\&task=view\&id=3820\&Itemid=2>. Acesso em: 28 maio 2020.

HAMMAL, F.; CHAPPELL, A.; WILD, T. C.; KINDZIERSKI, W.; SHIHADEH, A.; VANDERHOEK, A.; KHANH HUYNH, C.; PLATEEL, G.; FINEGAN, B. A. 'Herbal' but potentially hazardous: an analysis of the constituents and smoke emissions of tobacco-free waterpipe products and the air quality in the cafés where they are served. Tobacco Control, v. 24, n. 3, p. 290-297, London, 2015. Disponível em: <https://tobaccocontrol.bmj.com/content/24/3/290>. Acesso em: 28 maio 2020.

INCA. Instituto Nacional do Câncer. Tabagismo: um grave problema de saúde pública. Rio de Janeiro: Ministério da Saúde, $2007 . \quad$ Disponível em: <https://www.inca.gov.br/sites/ufu.sti.inca.local/files//media/document//tabagismo-grave-problema-desaude-publica-2007.pdf>. Acesso em: 28 maio 2020. 
INCA. Instituto Nacional do Câncer. Uso de narguilé: efeitos sobre a saúde, necessidades de pesquisa e ações recomendadas para legisladores. 2. ed. Rio de Janeiro: Ministério da Saúde, 2017. Disponível em: $<$ http://apps.who.int/iris/bitstream/handle/10665/161991/9789241508469-por.pdf?sequence $=5>$. Acesso em: 28 maio 2020.

MACIEL, Víctor. Estudo inédito aponta que 18,5\% dos adolescentes experimentam cigarro. Brasília, DF, 31 maio 2016. Disponível em: <https://www.gov.br/saude/pt-br/assuntos/noticias/estudo-inedito-aponta-que18-5-dos-adolescentes-experimentam-cigarro>. Acesso em: 28 maio 2020.

MAKIAK, W. The global epidemic of waterpipe smoking. Addictive Behaviors, v. 36, n. 1-2, p. 1-5, Amsterdam, 2011. Disponível em: <https://doi.org/10.1016/j.addbeh.2010.08.030>. Acesso em: 28 maio 2020.

MAKIAK, W.; WARD, K. D.; EISSENBERG, T. Interventions for waterpipe smoking cessation. Cochrane Database of Systematic Reviews, v. 4, Hoboken, NJ, 2007. Disponível em: <https://doi.org/10.1002/14651858.CD005549.pub2>. Acesso em: 28 maio 2020.

MALCON, M. C.; MENEZES, A. M. B.; CHATKIN, M. Prevalência e fatores de risco para tabagismo em adolescentes. Revista de Saúde Pública, v. 37, n. 1, p. 1-7, São Paulo, 2003. Disponível em:

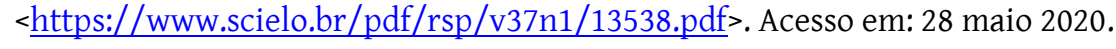

MALTA, D. C.; HALLAL, A. L. C.; MACHADO, I. E.; PRADO, R. R. do; OLIVEIRA, P. P. V. de; CAMPOS, M. O.; SOUZA, M. de F. M. de. Fatores associados ao uso de narguilé e outros produtos do tabaco entre escolares, Brasil, 2015. Revista Brasileira de Epidemiologia, v. 21, p. e180006, São Paulo, 2018. Disponível em: $<$ https://www.scielo.br/pdf/rbepid/v21s1/1980-5497-rbepid-21-s1-e180006.pdf>. Acesso em: 28 maio 2020.

MCDONALD, J. H. G-test of goodness-of-fit. 3. ed. Baltimore: Sparky House Publishing, 2014.

MENEZES, A. H. R.; DALMAS, J. C.; SCARINCI, I. C.; MACIEL, S. M.; CARDELLI, A. A. M. Fatores associados ao uso regular de cigarros por adolescentes estudantes de escolas públicas de Londrina, Paraná, Brasil. Cadernos de Saúde Pública, v. 30, p. 774-784, Rio de Janeiro, 2014. Disponível em: <http://www.biostathandbook.com/gtestgof.html>. Acesso em: 28 maio 2020.

NASCIMENTO, D.; SOARES, E. A.; FEITOSA. S.; COLARES, V. O hábito do tabagismo entre adolescentes na cidade de Recife e os fatores associados. Revista Odonto Ciência, v. 20, n. 50, p. 348-353, Porto Alegre, out./dez. 2005. Disponível em: <http://revistaseletronicas.pucrs.br/ojs/index.php/fo/article/viewFile/1184/943>. Acesso em: 28 maio 2020.

NOGUEIRA, K. T.; FUMO, C. M.; SILVA, M. Tabagismo em adolescentes numa escola de rede pública do estado do Rio de janeiro. Revista Adolescência e Saúde, v. 1, n. 4, p. 6-10, Rio de Janeiro, out./dez. 2004. Disponível em: $<$ https://cdn.publisher.gn1.link/adolescenciaesaude.com/pdf/v1n4a02.pdf>. Acesso em: 28 maio 2020.

OLIVEIRA, C. M. de. Fatores Motivacionais relacionados ao início do Tabagismo em estudantes adolescentes de Ribeirão Preto/SP. 2009. Dissertação (Mestrado em Ciências) - Universidade de São Paulo, Ribeirão Preto, 2009. Disponível em: <https://teses.usp.br/teses/disponiveis/59/59137/tde-14012010114209/publico/Cassiana.pdf>. Acesso em: 28 maio 2020. 
OMS. Organização Mundial da Saúde. Tabagismo \& Saúde nos Países em Desenvolvimento. Tradução Ministério da Saúde/Instituto Nacional do Câncer. Brasília: INCA, 2003. Disponível em: <http://bvsms.saude.gov.br/bvs/publicacoes/inca/tabagismo_saude.pdf/>. Acesso em: 10 jun. 2020.

ONUBR. Organização das Nações Unidas. OMS: 1 em cada 5 pessoas no mundo fuma. Brasília, 1ํjun. 2018. Disponível em: <https://nacoesunidas.org/oms-1-em-cada-5-pessoas-no-mundo-fuma/amp/>. Acesso em: 10 jun. 2020.

PASQUALOTTI, A.; MIGOTT, A. M. B.; MACIEL, E. N.; BRANCO, M. M. N.; CARVALHO, R. M. A.; PIZZOL, T. S. D.; GEHLEN, Carolina T.; SOLDA; Daiane A.; GRESSLER, Marina. Experimentação de fumo em estudantes do ensino fundamental e médio da área urbana da região Sul do Brasil. Revista Interamericana de Psicología, v. 40, n. 2, p. 213-218, Porto Alegre, 2006. Disponível em: <http://pepsic.bvsalud.org/pdf/rip/v40n2/v40n2a09.pdf>. Acesso em: 25 fev. 2006.

PASSOS, M. A. N.; DA CONCEIÇ̃̃o OLIVEIRA, A. Efeitos do uso do narguilé sobre a saúde e a visão dos jovens sobre o tema. Revista JRG de Estudos Acadêmicos, v. 2, n. 4, p. 165-171, Brasília, 2019. Disponível em: <http://revistajrg.com/index.php/jrg/issue/view/4>. Acesso em: 25 fev. 2021.

PETO, R.; DARBY, S.; DEO, H.; SLICOCKS, P.; WHITLEY, E.; DOLL, R. Smoking, smoking cessation, and lung cancer in the UK since 1950: combination of national statistics with two case-control studies. British Medical Journal, v. 321, p. 323-329, London, 2000. Disponível em: <https://www.bmj.com/content/bmj/321/7257/323.full.pdf>. Acesso em: 10 jun. 2020.

PINTO, M.; BARDACH, A.; PALACIOS, A.; BIZ, A.; ALCARAZ, A.; RODRIGUEZ, B.; AUGUSTOVSKI, F.; PICHONRIVIERE, A. Carga do tabagismo no Brasil e benefício potencial do aumento de impostos sobre os cigarros para a economia e para a redução de mortes e adoecimento. Cadernos de Saúde Pública, v. 35, p. e00129118, Rio de Janeiro, 2019. Disponível em: <https://www.scielo.br/pdf/csp/v35n8/1678-4464-csp-35-08-e00129118.pdf>. Acesso em: 30 maio 2020.

POLETTO. L.; PEZZOTTO, S. M.; MORINI, J.; ANDRADE, J. Prevalencia del habito de fumar en jóvenes y sus padres. Asociaciones relevantes con educación y ocupación. Revista de Saúde Pública, v. 25, p. 388-393, São Paulo, 1991. Disponível em: <https://www.scielo.br/pdf/rsp/v25n5/11.pdf>. Acesso em: 30 maio 2020.

$\mathrm{R}$ CORE TEAM. R: language and environment for statistical computing. $\mathbf{R}$ Foundation for Statistical Computing, Vienna: R Core, 2021. Disponível em: <https://www.gbif.org/pt/tool/81287/r-a-language-andenvironment-for-statistical-computing>. Acesso em: 30 maio 2020.

REVELES, C. C.; SEGRI, N. J.; BOTELHO, C. Fatores associados à experimentação do narguilé entre adolescentes. Jornal de Pediatria, v. 89, n. 6, p. 583-587, Porto Alegre, 2013. Disponível em: $<$ https://www.scielo.br/pdf/jped/v89n6/v89n6a11.pdf>. Acesso em: 10 jun. 2020.

SAITO, M. I. Adolescência, cultura, vulnerabilidade e risco. Pediatria, v. 22, n. 3, p. 217-219, Säo Paulo, 2000.

SANTOS, J. D. F.; SILVEIRA, D. V.; OLIVEIRA, D. F.; CAIAFFA, W. T. Instrumentos para avaliação do tabagismo: uma revisão sistemática. Ciência \& Saúde Coletiva, v. 16, n. 12, p. 4707-4720, Rio de Janeiro, dez. 2011. Disponível em: <https://www.scielo.br/pdf/csc/v16n12/20.pdf>. Acesso em: 20 abr. 2020. 
SCHNEIDER, A. R. dos S.; ALVES, G. G. Tabaco e adolescentes: a promoção da saúde no contexto da integralidade. Cadernos Saúde Coletiva, v. 17, n. 4, p. 1059-1073, Rio de Janeiro, 2009. Disponível em: $<$ http://www.cadernos.iesc.ufrj.br/cadernos/images/csc/2009_4/artigos/Artigo_9.pdf>. Acesso em: $20 \mathrm{abr}$. 2020.

SHIHADEH, A.; SCHUBERT, J.; KLAIANY, J.; EL SABBAN, M.; LUCH, A.; SALIBA, N. A. Toxicant content, physical properties and biological activity of waterpipe tobacco smoke and its tobacco-free alternatives. Tobacco Control, v. 24, Suppl 1, p. i22-i30, London, 2015. Disponível em: <https://pubmed.ncbi.nlm.nih.gov/25666550/>. Acesso em: 20 abr. 2020.

TURNER, L.; MERMELSTEIN, R.; FLAY, B. Individual and contextual influences on adolescent smoking. In: DAHL, R. E.; SPEAR, L. P. (ed.). Annals of the New York Academy of Sciences. v. 1021. New York: Wiley-Blackwell; Academy of Sciences, 2004.p. 175-197. Disponível em: <https://nyaspubs.onlinelibrary.wiley.com/doi/abs/10.1196/annals.1308.023>. Acesso em: 20 abr. 2020.

VICENTE, P. Prevalência e análise do tabagismo em adolescentes. 2010. Dissertação (Mestrado em Análise do Comportamento) - Universidade Estadual de Londrina, Londrina, 2010. Disponível em: <http://www.uel.br/pos/pgac/wp-content/uploads/2014/03/Preval\%C3\%AAncia-e-an\%C3\%A1lise-dotabagismo-em-adolescentes.pdf $>$.

VIEIRA, P. C.; AERTS, D. R. G. C.; FREDDO, S. L.; BITTENCOURT, A.; MONTEIRO, L. Uso de álcool, tabaco e outras drogas por adolescentes escolares em município do Sul do Brasil. Cadernos de Saúde Pública, v. 24, n. 11, p. 2487-2498, Rio de Janeiro, nov. 2008. Disponível em: <https://www.scielo.br/pdf/csp/v24n11/04.pdf>. Acesso em: 11 set. 2020.

World Health Organization. Report on the global tobacco epidemic, 2008: the MPOWER package. Geneva: WHO, 2008.2 Disponível em: <http://apps.who.int/iris/bitstream/handle/10665/43818/9789241596282_eng.pdf?sequence=1>. Acesso em: 30 maio 2020.

WORSNOP, C. Smoking: not for anyone. Chest, v. 123, p. 1338-1340, Glenview, Il, 2003. Disponível em: $<$ https://journal.chestnet.org/action/showPdf?pii=S0012-3692\%2815\%2933659-X>. Acesso em: 30 maio 2020. 


\section{ANEXO 1 \\ Questionário sobre tabagismo}

1. Idade:

2. Sexo
( ) Feminino
( ) Masculino

3. Escola em que estuda:

4. Qual ano você está cursando atualmente no Ensino Médio?
( ) $1^{\circ}$ ano
( ) $2^{\circ}$ ano
( ) $3^{\circ}$ ano

5. Você tem ou já teve interesse em fumar cigarro, narguilé ou cigarro eletrônico/vape?
( ) Nunca tive interesse
( ) Já tive interesse, mas não tenho mais
( ) Já tive interesse e fumei
( ) Tenho interesse e gostaria de experimentar

6. Você já fumou cigarro?
( ) $\operatorname{Sim}$
( ) Não

7. Com qual a idade você experimentou cigarro pela primeira vez?
( ) 10 anos ou menos
( ) 11 a 15 anos
( ) 16 a 20 anos
( ) Nunca experimentei

8. Qual a frequência do uso de cigarro durante a semana?
( ) 1 a 5 vezes na semana
( ) 6 a 10 vezes na semana
( ) 11 ou mais vezes na semana
( ) Nenhuma vez na semana

9. Você já fumou narguilé?
( ) $\operatorname{Sim}$
( ) Não 
10. Qual a frequência do uso de narguilé durante a semana?
( ) 1 a 5 vezes na semana
( ) 6 a 10 vezes na semana
( ) Mais de 10 vezes na semana
( ) Nenhuma vez na semana

11. Com qual a idade você experimentou narguilé pela primeira vez?
( ) 10 anos ou menos
( ) 11 a 15 anos
( ) 16 a 20 anos
( ) Nunca experimentei

12. Você já fumou cigarro eletrônico e/ou vape?
( ) $\operatorname{Sim}$
( ) Não

13. Qual a frequência do uso de cigarro eletrônico e/ou vape durante a semana?
( ) 1 a 5 vezes na semana
( ) 6 a 10 vezes na semana
( ) 11 ou mais vezes na semana
( ) Nenhuma vez na semana

14. Com qual a idade você experimentou cigarro eletrônico e/ou vape pela primeira vez?
( ) 10 anos ou menos
( ) 11 a 15 anos
( ) 16 a 20 anos
( ) Nunca experimentei

15. Qual a principal influência para você ter começado a fumar?
( ) Amigos fumantes
( ) Familiares fumantes
( ) Mídia
( ) Não fumo

16. Você tem conhecimento que o narguilé é uma forma diferenciada do uso do tabaco?
( ) $\operatorname{Sim}$
( ) Não

17. O que faz você ter vontade de fumar? (É permitido assinalar mais de uma opção)

( ) Ver amigos fumando 

( ) Ver familiares fumando
( ) Fuma por vontade própria
( ) Não fuma

18. O uso de bebida alcoólica faz você ter vontade de fumar?
( ) $\operatorname{Sim}$
( ) Não

19. Você já notou algum sintoma decorrente do tabagismo (Ex: tosse, falta de ar, entre outros)?
( ) $\operatorname{Sim}$
( ) Não

20. Você já teve alguma informação sobre o uso do tabaco? (Palestras em escolas, comerciais na televisão, mídia etc.).
( ) $\operatorname{Sim}$
( ) Não

21. A que situações o tabagismo está associado no seu dia-a-dia? (É permitido assinalar mais de uma opção)

( ) Com bebidas alcoólicas/outros tipos de bebidas (Ex: café)

( ) Ansiedade/tristeza

( ) Alegria

( ) Nenhum

( ) Não fumo

( ) Outros:

\section{(cc) $\mathbf{E Y}$}

Este trabalho está licenciado com uma Licença Creative Commons - Atribuição 4.0 Internacional. 